\title{
LAS SOFTAREAS Y EL TRABAJO INDEPENDIENTE CON EL EMPLEO RECURSOS TECNOLÓGICOS
}

\section{SOFTWARE AND INDEPENDENT WORK WITH EMPLOYMENT TECHNOLOGICAL RESOURCES}

\section{GEILERT DE LA PEÑA CONSUEGRA', GEMA ZAMORA LOOR², JOSÉ GILER SARMIENTO³, MARCOS VINCES CENTENO}

1 Universidad de La Habana, Cuba. gmilenium2017@gmail.com

2 Universidad Técnica de Manabí, Ecuador.jairo_vinces@gmail.com

3 Universidad Técnica de Manabí, Ecuador. jagiler@gutm.edu.ec

$4 \quad$ Universidad Técnica de Manabí, Ecuador. marcosvincescenteno@gmail.com

RESUMEN

Es el trabajo independiente desde y con el uso de los recursos tecnológicos, una posibilidad de lograr la elevación del aprendizaje como consecuencia de la motivación y uso de medios, en la búsqueda de la eficiencia y eficacia en las clases contemporáneas. En el estudio realizado, se tuvo en cuenta el trabajo independiente a partir del empleo de herramientas y recursos tecnológicos, en relación con los contenidos de asignaturas del 2do años de las cinco carreras de la Facultad de Ciencias de la Salud de la Universidad Técnica de Manabí. Para contribuir al desarrollo exitoso de este proceso, en el estudio realizado, se diseñaron un conjunto de softareas para implementarse en el trabajo independiente a realizar por los estudiantes, posterior a un diagnóstico caracterizador de los docentes sobre el nivel de conocimiento y funcionalidad de softareas orientadas por ellos. Además, para este último, es de vital importancia la reflexión teórico-metodológica asumida para la estructuración, orientación, ejecución y evaluación de dichas softareas. Estas tareas con software 0 softareas, potencian la motivación del estudiante y del docente en el proceso de enseñanza aprendizaje de las diversas materias, al elevar con resultados positivos, los índices de apropiación de conocimientos en tal sentido. Sobre la base de los resultados obtenidos a través de los diferentes métodos y técnicas empleadas, se pudo conocer la necesidad de uso y pertinencia de las softareas, y constatar las potencialidades de la propuesta, mediante su implementación en la práctica pedagógica.

PALABRAS CLAVE: recursos informáticos; software; softareas; trabajo independiente.
ABSTRACT

It is the independent work from and with the use of technological resources, a possibility of achieving the elevation of learning as a consequence of the motivation and use of means, in the search for efficiency and effectiveness in contemporary classes. In the study carried out, independent work was taken into account based on the use of technological tools and resources, in relation to the contents of subjects of the 2nd year of the five careers of the Faculty of Health Sciences of the Technical University of Manabí. To contribute to the successful development of this process, in the study carried out, a set of softareas were designed to be implemented in the independent work to be carried out by the students, after a characterizing diagnosis of the teachers on the level of knowledge and functionality of oriented softareas for them. In addition, for the latter, the theoreticalmethodological reflection assumed for the structuring, orientation, execution and evaluation of these softareas is of vital importance. These tasks with software or softareas, enhance the motivation of the student and the teacher in the teaching-learning process of the various subjects, by raising, with positive results, the rates of knowledge appropriation in this regard. Based on the results obtained through the different methods and techniques used, it was possible to know the need for the use and relevance of the softareas, and to verify the potential of the proposal, through its implementation in pedagogical practice.

KEYWORDS: computer resources; software; softareas; independent work. 


\section{INTRODUCCIÓN}

Las transformaciones que se realizan de forma constante en los diferentes modelos educativos a nivel mundial exigen de un perfeccionamiento sistemático y continuo en la labor de los docentes en aras de elevar la calidad de la enseñanza y el aprendizaje, lo cual, se han visto favorecidos con la introducción de las tecnologías educativas (Quiroz, \& Quiroz, 2019). De ahí, la necesidad de buscar nuevas vías que permitan desarrollar las capacidades y habilidades de los estudiantes para enfrentarse a las disímiles exigencias contemporáneas, en la cual, la independencia y la creatividad del individuo son más necesarios.

En el estudio en cuestión, se le concede gran interés a la necesidad de elevar el nivel de preparación teórica y metodológica en los docentes de las carreras de la Universidad Técnica de Manabí, para encausar adecuadamente la dirección, planificación, orientación y control del trabajo independiente en los estudiantes, a partir del uso de la virtualización y los diversos dispositivos electrónicos, como herramienta indispensable para lograr tal exigencia.

El desarrollo del trabajo independiente(Paz, León, Travieso, Martínez, \& Labrador, 2016), como componente del proceso de enseñanza aprendizaje en cualquier disciplina y nivel educativo, siempre ha constituido prioridad en el perfeccionamiento del sistema educacional en general, para algunos se entiende, como el trabajo autónomo, el cual, se coincide en la trascendencia de la independencia cognoscitiva de los estudiantes y su aprendizaje.

Al margen de todo lo que se ha logrado en este quehacer, existen regularidades detectadas en el diagnóstico fáctico realizado, a través de las visitas a clases, la revisión de cuadernos a los estudiantes, en el control de las actividades metodológicas y de capacitación que se realizan a los docentes y en la experiencia acumulada por los profesores que se relacionan con el presente estudio. Estos evidenciaron las siguientes limitaciones:

- Insuficiente nivel de preparación mostrada por los docentes para diseñar las actividades independientes con el uso de los recursos informáticos.

- Insuficiente actividades concebidas por los docentes para el desarrollo del trabajo independiente en diversas asignaturas del 2do año de diversas carreras de la Facultad de Ciencias de la salud de la Universidad Técnica de Manabí (UTM).
- Las actividades independientes planificadas y orientadas por los docentes, no revelan las potencialidades del uso de los recursos informáticos para elevar la calidad de los procesos de enseñanza aprendizaje.

- En el diseño y ejecución de las tareas de carácter independiente vinculadas con el uso de diversos recursos tecnológicos y dispositivos, predomina el nivel reproductivo y generalmente no se propicia la reflexión en el estudiante.

- En las tareas planificadas para el trabajo independiente, no se concibe suficientemente la unidad de los componentes intelectuales y motivacionales de la actividad cognoscitiva del estudiante.

Aunque diversos han sido los esfuerzos de los docentes que dirigen, orientan y favorecen los procesos de enseñanza-aprendizaje, por incentivar la actividad independiente de los estudiantes, aún persisten dificultades relacionadas con el predominio en las aulas de un proceso esencialmente instructivo, cognoscitivo (Mejías, Acosta, Andrés, Dueñas, \& Enmanuel, 2017), en el cual se centran las acciones mayormente del docente y en menor medida en el estudiante.

El estudiante tiende a aprender de forma reproductiva, afectándose el desarrollo de habilidades y de sus posibilidades para la reflexión crítica y autónoma de la construcción de los conocimientos, de ahí, que su inclusión consciente en el proceso, en ocasiones se encuentre limitado.

Al analizar estas dificultades, se apunta a una inadecuada dirección, planificación y orientación por el docente del trabajo independiente de los estudiantes, en insuficiencias en el empleo de herramientas y recursos tecnológicos, de manera que esta no estimula ni propicia el desarrollo de la independencia cognoscitiva de los estudiantes, en la búsqueda, procesamiento y transformación de la información.

\section{DESARROLLO}

Las concepciones y tendencias que sobre trabajo independiente han abordado los investigadores y pedagogos, para lograr la independencia cognoscitiva del estudiante en su quehacer investigativo son múltiples. Para su comprensión es necesario realizar el correspondiente análisis en cuestión, la cual se contextualiza desde el procesamiento de la información durante la clase, hasta llevar a cabo un proyecto de trabajo eficiente y eficaz. 
El trabajo independiente a cumplimentar por parte de los estudiantes, y su concepción a partir de los diferentes medios utilizados para su desarrollo, poseen una cantidad considerable de antecedentes en la teoría pedagógica. Condicionado este por la unidad de la instrucción y la educación, que posibilita el perfeccionamiento del mismo.

Resulta importante destacar que, diversos son los criterios de los autores consultados relacionados con las definiciones existentes sobre el trabajo independiente. Se considera importante destacar, lo enunciado por Yesipov (1981), quién considera el trabajo independiente como.

“(...) aquel que se realiza sin la participación directa del maestro, pero por orientación del mismo, en un tiempo establecido y durante el cual los estudiantes se esfuerzan conscientemente por lograr los objetivos planteados, manifestando de una forma u otra, los resultados de su actividad física o mental, o ambas (...)”. (Yesipov, 1981 p. 21)

En el análisis realizado a los criterios de los autores antes mencionados y otros, son de vital importancia a criterio del autor los ofrecidas por Pidkasisti, (1986), y Álvarez de Zayas, (1999). El primero lo considera el trabajo independiente como “(...) el medio de inclusión de los estudiantes en la actividad cognoscitiva independiente, el medio de su organización lógica y psicológica (...)" (Pidkasisti, 1986 p. 67), y el segundo lo generaliza como, “(...) el modo de organización del proceso docente, dirigido a la formación de la independencia, como característica de la personalidad del estudiante" (Álvarez de Zayas, 1999 p. 44). Se destaca como aspecto común, el hecho de considerar el trabajo independiente como el modo de organización de la actividad cognoscitiva del estudiante en el proceso docente - educativo. (Fuente, García, \& Rodríguez, 2016).

Son significativos los aportes logrados sobre trabajo independiente que referencian otros autores, en relación al grado de complejidad con que debe ser concebida la actividad independiente.

Se plantea, que el trabajo independiente se expresa desde la siguiente tipología:

1. Trabajo independiente de reproducción según el modelo.

2. Trabajo independiente de reconstrucción.

3. Trabajo independiente heurístico (o de variación).
4. Trabajo independiente de creación (investigativos).

En consideración con lo abordado, se puede caracterizar el trabajo independiente al atender la clasificación ofrecida por este autor.

1. Trabajo independiente reproductivo.

2. Trabajo independiente productivo.

3. Trabajo independiente creativo.

El carácter productivo brinda la presencia del análisis, síntesis, inducción, deducción, comparación, abstracción, generalización, entre otros procesos mentales, en la solución de problemas, o en la concreción de tareas retadoras en las cuales la aplicación de los conocimientos adquiridos por el estudiante se hacen necesarios. Por último, el trabajo independiente creativo depende de las habilidades investigativas del estudiante en la búsqueda de soluciones a tareas o problemas planteados.

La actividad debe proyectarse de manera que permita el papel activo y reflexivo del estudiante, se organiza a partir de la posibilidad de interacción, como momento inicial en que aparecen los procesos psicológicos, los desempeños y competencias cognitivas.

Las actividades independientes o tareas independientes ofrecen la oportunidad del intercambio entre los sujetos.

Álvarez de Zayas, (1999), le atribuye a la tarea docente un carácter personológico al afirmar que en relación a la misma, el proceso de enseñanza - aprendizaje se individualiza, al ser el estudiante el centro de la tarea. Criterios que se comparten, al igual que sostiene, que en cada tarea hay un conocimiento a asimilar, una habilidad a desarrollar y valores a formar. Explica, que cuando la tarea se plantea debidamente, permite que los estudiantes aporten todas sus ideas, inclinaciones e impresiones para darle solución a la misma, y por ende contribuye al desarrollo de la personalidad de forma integral.

Los anteriores criterios, son aportaciones sobre la actividad creadora de los estudiantes, que contribuye a una mejor comprensión de la concepción de la formación desarrolladora en los estudiantes, desde el proceso de enseñanzaaprendizaje. En consideración la estructuración didáctica de la tarea se concibe a partir del vínculo entre su contenido teórico y la realidad educativa, por lo que, su solución implica la realización de acciones investigativas tales como: 
- Observar la realidad.

- Elaborar y aplicar instrumentos de investigación.

- Realizar búsquedas bibliográficas.

- Utilizar recursos informáticos.

- Valorar criterios científicos.

- Comparar puntos de vistas de diferentes autores.

- Comparar la teoría y la práctica.

- Asumir posiciones personales en el debate científico.

- Proyectar alternativas de solución a los problemas.

- Comunicar de forma oral y/o escrita los resultados del trabajo.

Para lograr la concepción de la tarea independiente, es menester asimilar los conocimientos mediante acciones necesarias a partir de su estructura, que según Soca Guevara, 2015), se concreta en:

a. La parte orientadora.

b. La parte ejecutora.

c. La parte de control.

En correspondencia con las TIC que se configuran como significativos avances en el campo pedagógico. Estas convergen en los entornos educativos e investigativos de los diferentes niveles educacionales, y se impone en gran medida la necesidad de elevar la preparación cultural, tecnológica y educativa.

Marquéz, (2001), plantea: “(...) estos símbolos están siendo referenciados por las nuevas Tecnologías de la Información y las Comunicaciones (...)" (Marquéz, 2001 p. 89), este lo expone como la vía moderna de un aprendizaje, a partir de sistemas de símbolos que son contenido de las tecnologías emergentes.

En esta dirección, el uso de las TIC posibilita que el profesor se enfrente a un nuevo rol, más profesional, creativo y autónomo, que le permite aprovechar las tecnologías tanto para la enseñanza como para su propio aprendizaje permanente. (Ramos, Domínguez, Gavilondo, \& Fresno, 2008).

En relación con este aspecto, el docente debe buscar alternativas que generen una interacción eficaz entre los estudiantes y su entorno. Igualmente Campos, (2014) en su material "El aula sin frontera”, hace referencia a que “(...) quienes tienen acceso a estas tecnologías, no pueden dejar de reconocer las múltiples ventajas que traen aparejadas en todos los órdenes del quehacer humano y en sus entornos sociales (...)" (Campos, 2014 p. 115), pero también se enfrenta al desafío de incorporar, a través de la exploración y la creación, nuevas metodologías de enseñanzas a partir del adecuado uso de estos medios tecnológicos.

En consideración con esto criterios, se comprende que la incorporación de herramientas y recursos tecnológicos en el proceso de enseñanza aprendizaje permite que:

- El profesor conozca las potencialidades y diferentes posibilidades del empleo de este medio, e identifique las situaciones en las que se hace un uso inteligente de la misma.

- Se evalúe críticamente los alcances de las TIC, las herramientas y recursos tecnológicos específicos y factibles, así como su impacto en la institución universitaria.

- Se utilice el recurso, como una herramienta más de trabajo, una opción que favorece el autoaprendizaje en los estudiantes.

Desde este punto de vista, se conoce que en los salones de clases existen recursos y medios novedosos para comunicarse e interactuar, para investigar y publicar, para debatir y compartir, independientemente del espacio y el tiempo. Esto exige de una mayor exigencia en función de la autopreparación y actualización, lo que resulta en un aprendizaje y operacionalización de las nuevas tecnologías y sus recursos informáticos. Esto se expresa en la suposición lógica de que si la oferta es amplia y enriquecida, disponen por lo tanto de medios para enseñar y profundizar en un aprendizaje eficiente en los estudiantes.

Es recurrente pero necesario significar el uso inteligente con los que deben ser tratado los recursos tecnológicos como apoyo al proceso de enseñanza aprendizaje. Que responda a los intereses y necesidades cognitivas de los estudiantes y a una enseñanza desarrolladora, y así permitir su formación integral, desde el estudio independiente como un espacio de interacción social, donde el estudiante se apropie de los conocimientos a partir del apoyo de los recursos tecnológicos.

Desde esta óptica se posibilita la constitución y se contribuya con un medio imprescindible en el marco de los nuevos ambientes de aprendizaje, para optimizar de esta forma la actividad y la 
comunicación de los docentes con los estudiantes, y de estos entre sí. Uno de estos ambientes de aprendizaje lo constituye a partir del empleo de herramientas y recursos tecnológicos, y software especializados en mayor medida, que según Coloma, (2003), lo asume como una: "(...) aplicación informática que puede ser utilizada como medio de enseñanza en el proceso de enseñanza aprendizaje" (Coloma, Salazar, \& González, 2003 p. 6). Además, considera que su uso, puede fomentar cualidades de la personalidad o favorecer la utilización de procedimientos y estrategias mentales necesarias para dicho aprendizaje. Delgado, Coloma, \& Salazar, 2014).

Por su carácter interactivo y la capacidad de individualizar el proceso, más que un medio de enseñanza debería llamarse medio de aprendizaje, pues el hecho de estar basado en tecnología hipermedial destaca entre sus ventajas, como plantea Rodríguez, \& Caro, (2003):

- Facilidad para moverse (navegar) sobre la información.

- Lectura (consulta) del documento adaptado al usuario.

- Permite enlazar textos con imágenes, sonidos, videos.

- Permite elevar la interacción hombre máquina.

- Logra en determinados momentos efectos que no son posibles lograr en clase con otros medios de enseñanza, tales como representar el comportamiento de los diferentes cuerpos en el espacio, situación que para lograrla es necesario apelar a la abstracción del estudiante.

- Otra ventaja que brinda, es la de obtener una mayor motivación para el estudio, así como lograr con el sonido y la imagen explicaciones de los diferentes temas a tratar en el software.

En consecuencia con estos aspectos, es considerable poner en función del trabajo independiente, los recursos informáticos, al considerar que resulta propicio en este componente del proceso de enseñanza aprendizaje, para el incremento del nivel de independencia cognoscitiva del estudiante. Le permite al profesor tomar decisiones sobre las tareas que debe planificar y orientar a los estudiantes en correspondencia con su nivel de desarrollo, de acuerdo con sus posibilidades reales de aprendizaje.

El éxito de una enseñanza desarrolladora en los estudiantes, depende en gran medida, no sólo del grado de desarrollo de los recursos cognitivos que le permiten un pensamiento creativo, sino también del interés que muestre el alumno por la tarea, el grado de satisfacción y motivación. Así como también, el nivel de desarrollo intelectual e investigativo en relación con sus principales gustos, preferencias y aspiraciones.

El uso que un estudiante puede hacer de una herramienta o recurso tecnológico (Sánchez, Maden, \& Blázquez, 2018) para garantizar su estudio independiente, destaca Chala, (2006) es muy amplio. Se plantea que diversos son los programas que pueden ser considerados como recursos tecnológicos para el desarrollo de la actividad independiente, los cuales comprenden cualquier "Aplicación Informática que utiliza una estrategia de aprendizaje, constituye un medio efectivo de enseñanza" (Mena, 2006 p. 276), para desarrollar el trabajo independiente, y de esta forma se propician beneficios en ahorro de tiempo, confiabilidad en los resultados, ahorro de esfuerzo, productividad, entre otros de uso general o específico, que se pueden utilizar para una correcta concepción en el desarrollo del trabajo independiente con la tecnología.

Dentro del primer grupo, se encuentran los sistemas elaborados para hacer más dinámico y eficiente el trabajo diario. Programas que van encaminados a aumentar la productividad, eficiencia y eficacia de las personas. Entre estas están los siguientes:

- Procesadores de textos, que tienen como finalidad general la elaboración de materiales y trabajos escritos.

- Procesadores gráficos, los que han permitido que la expresión gráfica se multiplique.

- Procesadores numéricos, encaminados al manejo y procesamiento de grandes volúmenes de números.

- Procesadores musicales para la edición de sonidos.

- Manejadores de bases de datos, con la finalidad de procesar, analizar, almacenar, gestionar, entre otros, grandes volúmenes de información.

- Exploradores, que permiten el acceso del conocimiento desde lugares distantes a través de las redes de computadoras.

Permiten mejorar el entorno de aprendizaje. Por tanto, contribuir a la apropiación y desarrollo 
de habilidades, hábitos y capacidades investigativas, necesarias en su formación desarrolladora. Se utilizan dentro y fuera de la clase donde éste hace uso de ella, puede solucionar tipos de problemas cuyos resultados, a partir del proceso de búsqueda, selección, procesamiento interactivo de la información, permite la indagación e investigación y por ende, arribar a conclusiones.

El empleo inteligente y oportuno de todos estos programas o aplicaciones, contribuyen a favorecer el trabajo independiente, así como, facilitar el nivel de independencia de los estudiantes en la asimilación de los conocimientos, a partir de su correcta dirección, planificación, orientación y ejecución que permita una buena optimización en su uso. Desde este punto de vista, es considerable comprender que las softareas esencian esta generalidad en su estructura, así como, el tratamiento a diferentes contenidos sobre las base de la proyección interactiva con los recursos informáticos. (Ducasse, González, \& Laborí, 2015).

METODOLOGÍA

Desde el punto de vista metodológico se tienen en cuenta en tal sentido, que el estudio realizado fue descriptivo de corte experimental, en el cual, se utilizaron los siguientes métodos:

Histórico - lógico, Análisis - síntesis, Inducción - deducción, Observación científica, Encuesta, Entrevista.

Los instrumentos aplicados se utilizaron con los principales participantes relacionados con el proceso estudiado.

Para constatar el dominio que poseen los docentes, en todo lo relacionado con el proceso de dirección, planificación y orientación del trabajo independiente, en relación con el uso de los recursos tecnológicos y lograr softareas, se tuvieron en cuenta como población, los 19 docentes que trabajan en el 2do año de diversas carreras de la UTM, y 332 estudiantes de los paralelos focalizados. De estos, se seleccionaron 6 docentes y 32 de los estudiantes de diversas carreras, los que representan la muestra.

Se asume la perspectiva de dar salida al trabajo independiente en los procesos de enseñanza - aprendizaje de diversas asignaturas de las carreras de la UTM, desde la utilización eficaz y eficiente de los recursos tecnológicos existente. Desde esta visión, la novedad científica de la investigación está dada, a partir del conjunto de Softareas elaboradas para favorecer el trabajo independiente de los estudiantes, así como las potencialidades de estos recursos para elevar el aprendizaje de dichas asignatura. El conjunto de softareas (Silva, 2015) sirven de material de consulta para el docente, con función orientadora en la elaboración de otras de acuerdo con los contenidos que se trabajen en las diversas asignaturas. Además, permite la profundización necesaria del docente en el uso de la Tecnologías Educativas en el proceso docente (Médor, Rivas, Ramírez, 2018), que se revierte en una mayor preparación y nuevas vías para el perfeccionamiento de su labor.

\section{RESULTADOS Y DISCUSIÓN}

En particular, el empleo de los recursos, herramientas y dispositivos tecnológicos se ha convertido en un excelente medio y vía para los diversos procesos de enseñanza y aprendizaje, por su carácter interactivo y su capacidad de individualizar el proceso. Un ejemplo en este sentido, lo constituyen entre otros, el empleo de simuladores sobre las técnicas de diagnóstico de enfermedades oculares, así como el estudio del funcionamiento de estructuras oculares con software para ello, también el propio estudio el empleo de recursos educativos y materiales en la red que son de uso necesario para profundizar en los contenidos.

Por otra parte la sistematización de la reflexión teórico-metodológica asumida por los autores en la presente investigación, así como las softareas elaboradas, constituyen elementos de carácter orientador y práctico que te permite la concepción y materialización de futuras softareas en el resto de las unidades del sylabus, e incluso de otras asignaturas en la carrera. Cada tarea de aprendizaje, posee un nivel de ayuda desde el punto de vista del acceso a los recursos, de manera que permite la independencia del estudiante que aprende, en el desarrollo de las actividades que se programan u orientan.

Para obtener un diagnóstico acertado, de las principales insuficiencias presentadas por los docentes en la dirección, planificación y orientación del trabajo independiente, desde el uso de los recursos tecnológicos, se consideró una matrícula de 120 estudiantes aproximadamente en el 2do año de carreas de la UTM. Se tiene en cuenta en tal sentido, la cantidad de docentes en formación que trabajan con este año.

El estudio se realizó con una población de 19 docentes en general que imparten las asignaturas correspondientes. Se contó entre ellos, con 8 profesores especialistas con una amplia 
experiencia a tiempo completo, 11 docentes medio tiempo.

Se observaron clases desarrolladas por los docentes, para constatar evidencia del trabajo independiente desde el uso de software, herramientas y recursos tecnológicos y otros aspectos de carácter metodológico para su concepción. Así como, actividades de estudio para verificar el grado de desarrollo investigativo e intelectual y la independencia en las tareas orientadas por el docente. Se aplicaron encuestas a docentes que imparten las asignaturas y a estudiantes. Por último, se revisaron planes de clases y estrategias que se desarrollan en la actividad docente.

Además, se tuvo en cuenta en dicho diagnóstico, lo relacionado con los conocimientos que poseían sobre la concepción del trabajo independiente desde el uso de los recursos tecnológicos, el dominio de los software a utilizarse en las diversas asignaturas tratadas en el semestre, así como, el nivel de preparación en el empleo de la virtualización y las herramientas tecnológicas en tal sentido.

De esta forma, se obtuvieron resultados que constituyeron elementos a considera, para demostrar las limitaciones teóricas y metodológicas de los docentes en este componente del proceso de enseñanza aprendizaje. Estos principales resultados se relacionan a continuación:

Se les aplicó una entrevista a los profesores, la cual arrojó los siguientes resultados:

- El 61,1\% de los docentes reconocen el trabajo independiente, como componente indispensable del proceso de enseñanza aprendizaje, en el desarrollo de la independencia cognoscitiva del estudiante dentro del estudio, pero solo el 33,3\% conocen en qué bibliografía es abordado directamente desde lo metodológico en su desarrollo.

- Solo el $27,7 \%$ lograron clasificar el trabajo independiente según sus variantes, el resto no lo dominan.

- El 66,6\% no tienen en cuenta los aspectos metodológicos organizativos para planificar y diseñar un trabajo independiente desde el empleo adecuado de herramientas y recursos tecnológicos. Menos el 38,8\%, el resto de los docentes, no poseen un criterio lo suficientemente acertado y fundamentado de la importancia de la concepción desarrolladora que tiene en su aplicación el trabajo independiente.
- El $72,2 \%$ no saben realizar una correcta estructuración del trabajo independiente, a partir de las necesidades cognitivas y de aprendizaje del estudiante.

- El 66,6 \% conocen qué es un recurso tecnológico, pero solo el $27,7 \%$ reconoce los recursos tecnológicos que se pueden utilizar en el trabajo independiente y el desarrollo de los contenidos de las asignaturas.

- Sólo el 33,3 \% ha implementado los recursos tecnológicos en función del trabajo independiente, pero muy pocas veces, lo que pone de manifiesto las insuficiencias detectadas durante la primera etapa de la investigación.

Para determinar los principales factores que inciden en la concepción del trabajo independiente, desde el uso de los recursos tecnológicos en las asignaturas de la carrera en el 2do año, se realizó una encuesta a los docentes muestreados, donde esta arrojó los siguientes resultados:

- El $100 \%$ de los docentes opinan que en la asignatura que imparte se manifiestan todas las potencialidades de los contenidos, para desarrollar un correcto trabajo independiente por los estudiantes, con el criterio de que sería un trabajo muy bueno para la apropiación de todos los conocimientos concebidos en el syllabus.

- El 78,8\% de ellos, opinan que la planificación y orientación del trabajo independiente que realizan, desde el uso de los recursos tecnológicos no es el adecuado, pues según sus criterios, en reuniones de capacitación metodológica nunca se han desarrollados temas relacionado con la estructuración de actividades independientes en correspondencia con el uso de los recursos tecnológicos. En su generalidad, coinciden que no es suficiente la preparación metodológica recibida en este sentido, y es limitada la preparación que poseen para enfrentar las exigencias metodológicas, sobre la base del uso de estos.

- Solo el 26,6 \% considera correcta, la forma en que estructuran el trabajo independiente que orientan desde el punto de vista metodológico, a partir del uso de estos recursos. Por otra parte, se tienen diferentes criterios acerca de la estructura que debe llevar la softarea, 
para concebir el trabajo independiente de los estudiantes.

- El $100 \%$ consideran posible, formar una preparación especializada y general integral en los estudiantes a partir de la utilización de las Tecnologías de la Información y las Comunicaciones, por lo tanto, coinciden que es el medio idóneo para modelar aquellos fenómenos que por sus características y naturaleza, sería imposible estudiarlos en el salón de clase.

- Para advertir evidencias del trabajo independiente a partir del uso de determinados software y otros aspectos de carácter metodológico para su concepción, se observaron un total de 36 turnos de clases a 6 de los docentes de la muestra. Se constató, que no son sistemáticas las actividades independientes que se planifican y orientan para el trabajo con los software u otra aplicación informática. Se llega a la conclusión en tal sentido, que no hay dominio de esta metodología, ni se poseen las habilidades suficientes para la elaboración de las softareas. Pues al comprobar en 6 actividades observadas en su orientación, solo en el $33.3 \%$ de los docentes se manifiesta con claridad los objetivos propuestos, al propiciar el valor que posee el nuevo conocimiento para el aprendizaje.

- En el $66.6 \%$ de la muestra, dentro del tratamiento del contenido, predomina el trabajo independiente reproductivo.

- Con respecto a la concepción y orientación del trabajo independiente, en el $33.3 \%$ de las actividades observadas se tuvo en cuenta la motivación. En el 16.6 \% la estimulación de los procesos sensoperceptuales y en el $33.3 \%$, los procesos del pensamiento. En el $50.0 \%$ la unidad de los componentes intelectuales de la actividad cognoscitiva, en el resto no se trabaja con la atención a la diversidad que se requiere. En el $33.3 \%$, se pone de manifiesto el desarrollo de la memoria. En el $50.0 \%$ se logra la independencia cognoscitiva pero en ninguno de los casos, la creatividad de los estudiantes.

- Al analizar la estructura, con que estas actividades fueron diseñadas, se pudo constatar que en el $100 \%$, se utilizaron de alguna manera los recursos tecnológicos en la solución de las mismas.
- En el $50.0 \%$, se tuvo en cuenta el uso de algunas bibliografías. Solo en el $16.6 \%$, se analizaron documentos, pero en ninguno de los casos se valoraron criterios científicos, ni se compararon puntos de vistas de diferentes autores, lo que no se pudo asumir ninguna posición personal por parte de los estudiantes con respecto a ello. En el $16.6 \%$ se pudo proyectar alternativas de solución a los problemas, y en el $100 \%$ hubo comunicación escrita de los resultados del trabajo.

Para comprobar el grado de independencia con que los estudiantes ejecutan las diferentes actividades orientadas para el trabajo independiente a concretar durante las actividades de estudio presencial, se observaron las 6 actividades orientadas con antelación, arribándose a las siguientes conclusiones:

- En el $66.6 \%$ de las actividades, los estudiantes no conocen de antemano con qué herramientas se realizará la misma.

- En el $66.7 \%$ de las tareas, los estudiantes necesitan un nivel de ayuda significativo, por parte del docente, observándose solo en el $33.3 \%$ un determinado grado de independencia para ejecutar las actividades, lográndose culminar la actividad con éxito en el $50.0 \%$ de los estudiantes. Por tanto, se puede concluir que solo en el $50.0 \%$ de los casos se cumple el objetivo del trabajo independiente.

- El $100 \%$ de los estudiantes le atribuyen gran importancia al uso de los dispositivos y herramientas tecnológicas como herramienta de gran utilidad para dar solución a las diferentes actividades orientadas como trabajo independiente. Por otra parte, el $74.2 \%$, coinciden en que casi nunca el profesor orienta actividades a resolver con el empleo de medios y herramientas tecnológicas específicas a los contenidos que se trabajan.

Se plantean que se deben buscar nuevos métodos y estilos de trabajo que permitan, el tratamiento desde el punto de vista metodológico y exhaustivo de los contenidos, sobre todo con la ayuda y empleo de las tecnologías educativas.

Resultados obtenidos a partir de la instrumentación de la propuesta.

Se elaboró un material docente titulado "Conjunto de softareas para favorecer el trabajo 
independiente, a partir del uso de los recursos tecnológicos", fue instrumentado en el 2do año de la carrera de la UTM.

Para la instrumentación del mismo, se realizaron las siguientes actividades:

- Reunión metodológica o de capacitación por el investigador y especialistas en el empleo de los dispositivos y recursos tecnológicos en la docencia, dirigida a presentar oficialmente el material docente por los autores de la investigación.

- Se realizaron análisis valorativos de su contenido, y se trazaron las pautas para la instrumentación, con acuerdos a cumplimentar según dos etapas:

La primera etapa estuvo dirigida a la preparación de los docentes en el contenido del material docente.

- Actividad metodológica sobre la concepción del trabajo independiente desde el uso de los recursos tecnológicos en función de las Softareas.

- Clase práctica para interactuar con los diferentes recursos tecnológicos existentes, y explorar toda la información relacionada con los contenidos de las asignaturas desde el empleo de estos recursos y herramientas tecnológicas.

- Taller metodológico para diseñar softareas, al tener en cuenta los elementos teóricos metodológicos tratados en la primera actividad metodológica.

La segunda etapa está dirigida a poner en función los procesos de enseñanza - aprendizaje de las asignaturas que se desarrollaron en el primer semestre del 2do año de las carreras de la UTM, la preparación recibida por el docente durante la primera etapa de la instrumentación.

Una vez desarrolladas todas las acciones que intervienen en el proceso, se instrumentó la propuesta. En la fase final de dicha instrumentación, se procedió a la aplicación de una serie de instrumentos y técnicas de investigación, para la recogida de información.

Se aplicó la observación, la cual permitió obtener información durante el desarrollo de la clase. Con la intención de revelar las eviden- cias en la transformación de los docentes, en función de lograr una adecuada dirección, planificación, orientación y evaluación de las softareas desde el uso de software que lo posibilitan. Además, la aplicación de una entrevista, con el objetivo de obtener información sobre los conocimientos adquiridos, la aceptación del material instrumentado, y el nivel de preparación de los docentes para concebir y concretar una softarea. También, una encuesta a estudiantes, para comprobar el nivel de solución encontrado a las diferentes tareas orientadas por el docente.

Principales resultados obtenidos:

- En las observaciones a clases (40), se pudo apreciar un incremento en la planificación y orientación de nuevas softareas, así como, una adecuada preparación de los docentes desde el punto de vista teórico-metodológico para concebirlas.

- El $100 \%$ de los docentes llevaron a cabo con efectividad las diferentes etapas de preparación previa, de orientación de la softarea y control del cumplimiento de las actividades, y evidenciase en la calidad de estructura y diseño de la misma.

- El 90.0\% lograron elaborar tareas que respondieron a las exigencias intelectuales de los estudiantes, así como, a la utilización de variados recursos tecnológicos.

- El $100 \%$ de los docentes se rigieron por la estructura propuesta en el material, para el diseño de la softarea, lo que permitió el desarrollo de la independencia cognoscitiva de los estudiantes.

- Se evidenció la utilización de la bibliografía especializada en la concepción de algunas de las actividades planificadas en las softareas.

- Se logró un incremento en el aprovechamiento de los dispositivos y herramientas tecnológicas, para el estudio autónomo, el trabajo independiente en la elaboración de presentaciones y la profundización en los contenidos tratados, evidenciado por el interés y la motivación, para dar solución a las tareas con el empleo de ellas.

- Se revelaron resultados satisfactorios en el aprendizaje de los diferentes contenidos concebidos en cada softareas diseñada y utilizada, a partir de los mecanismos de búsqueda, procesamiento y transformación de la información. 
Lo anterior, derivado de la cantidad de tareas solucionadas por los estudiantes (61 de 67) como consecuencia de la indagación e investigación realizada por los estudiantes, a través de la utilización de los recursos informáticos.

- Los resultados de la entrevista, evidenciaron un alto nivel de motivación, así como significativa importancia que le conceden los docentes a los contenidos teóricos- metodológicos concebidos en la propuesta para concretar la softarea.

- La información obtenida a través de la observación de clases, se integró, ya que permitió revelar determinados elementos desde una posición interpretativa de lo acontecido en el desarrollo de las diferentes acciones y actividades planificadas para concebir las softareas.

Sin embargo, la entrevista fue necesaria aplicarla para profundizar en torno a los aspectos evaluados sobre la preparación del docente, la aceptación de las propuestas, sus criterios y recomendaciones al respecto. Dichos resultados se analizaron además, al tener en cuenta los datos obtenidos a partir de otras fuentes.

CONCLUSIONES

Se realizó un análisis teórico sobre la conceptualización del trabajo independiente a partir de criterios de otros investigadores, para su abordaje desde la concepción en función de la realidad del tema abordado.

Se encontró que las tendencias tradicionales de cómo enfocar el trabajo independiente de los estudiantes, con la introducción de las Tecnologías de la Información y la Comunicación, han carecido de elementos potenciadores de la actividad independiente, habilidades, capacidades y nuevos conocimientos. Al ser en tal sentido, limitadas en función del volumen de procesamiento y transformación de la información por parte del estudiante.

El diagnóstico desarrollado arrojó que en la estrategia de trabajo de los docentes en su preparación y desarrollo de las actividades de aprendizaje en sus asignaturas, no se incluyen acciones para dar tratamiento a la concepción del trabajo independiente desde el uso de los recursos tecnológicos, y esto evidencia la falta de preparación en este sentido.

El material docente elaborado, contiene el conjunto de softareas, y aborda elementos teóricos -metodológicos a tener en cuenta para diseñar y estructurar de las mismas, con los recursos tecnológicos con que se cuentan y se ajustan a los contenidos de las signaturas que se desarrollan. Esto se logra a partir de las tres etapas que el docente debe tener presente para su concreción.

En los estudiantes se revelaron transformaciones notables, en el orden de su independencia cognoscitiva y creadora. Reflejado a través del aprovechamiento óptimo de cada uno de los recursos tecnológicos previamente orientado por el docente. También en estos últimos, se evidenciaron avances en su nivel de preparación metodológica, al lograr concebir, el trabajo independiente sobre la base de los aspectos teórico -metodológicos contenidos en el material docente, y la adecuada profundización en lo relacionado con la elaboración, orientación, ejecución y evaluación de softareas.

Los criterios valorativos resultantes de la instrumentación de acciones efectuadas por los estudiantes y docentes en general, luego de puesto en práctica el material docente, fueron relevantes. Demostrándose a través de la aplicación de diversos métodos y técnicas que en las condiciones en que experimentó, son factible para obtener una notable transformación en los docentes, los cuales, se revierten en los resultados del aprendizaje de los estudiantes.

\section{REFERENCIAS BIBLIOGRÁFICAS}

Âlvarez de Zayas, C. (1999). La escuela en la vida. Ed. Pueblo y Educación. Ciudad de La Habana.

Campos, H. (2014). Objetos virtuales de aprendizaje en la formación tecnológica -aula sin fronteras para la formación de grumetes de segundo año de la tecnología naval en electrónica. Escenarios, 12(1), 111-117.

Coloma, O., Salazar, M., \& González, J. (2003). El software educativo en la clase ¿Intruso o aliado? Instituto Superior Pedagógico "José de la Luz y Caballero" En soporte electrónico., curso 69.

Delgado, F., Coloma, o. \& Salazar, M. (2014). La softarea en el proceso en el proceso de enseñanza - aprendizaje de la secundaria básica. Revista Didasc@lia: didáctica yeducación, 5(4).

Ducasse, N., González, A. \& Laborí, M. (2015). La softarea y sus potencialidades para el desarrollo de habilidades informáticas en la educación primaria. Estudios del desarrollo social: cuba y América Latina, 3 (1), 84-93.

Fuente, H., García, M. \& Rodríguez, Z. (2016). El trabajo independiente: su trascendencia en la 
formación de los profesionales de la medicina. MediSan, 20(12), 7022-7033.

Marquéz Grells, P. (2001). Algunas notas sobre el impacto de las TIC en la Universidad, educar, (28), 83-98.

Médor, D., Rivas, E. 6 Ramírez, M. (2018). Sobre las razones del emprendimiento y del trabajo independiente de los profesionales en tecnologías informáticas en Guadalaja, Jalisco. Trayectorias. Revista de ciencias sociales de la universidad autónoma de Nuevo León, 20(46), 93-118.

Mejías Gallegos, C., Acosta, M., Andrés, R., Dueñas, M. \& Enmanuel, D. (2017). El trabajo independiente: importancia en la calidad del proceso de enseñanza - aprendizaje en la carrera de odontología. Revista Universitaria y Sociedad, 9(2), 28-36.

Mena, A. (2006). La softarea, forma en que pueden usarse los software en el proceso docente-educativo. Mendiuve. Revista de educación, 4(4), 276-282.

Paz Paula, C., León Medina, D., Travieso Pérez, M., Martínez Carmona, Y. \& Labrador Falero, D. (2016). Nivel de conocimientos sobre el trabajo independiente de los profesores de la carrera de estomatología. Revista de Ciencia Médicas de Pinar del Río, 20(2), 81-89.

Pidkasisti, P. (1986). La actividad cognoscitiva de los alumnos en la enseñanza. La Habana.
Quiroz, D., \& Quiroz, M. (2019). Las tecnologías de la información y las comunicaciones (TICs) en la educación superior: consideraciones teóricas. REFCaIE. Revista Electrónica Formación y Calidad Educativa. ISSN. 1390-9010, 7(1), 213-228.

Ramos Pérez, L., Domínguez Lovaina, J., Gavilondo Mariño, X., \& Fresno Chávez, C. (2008). ¿Software educativo, hipermedia o entorno educativo? Acimed, 18(4), 0-0.

Rodríguez, A. \& Caro, E. (2003). Estilos de aprendizaje y e-learning. Hacia un mayor rendimiento académico. Revista de educación a distancia, (7).

Sánchez, R., Maden, A. \& Blázquez, R. (2018). Actividades de aprendizaje mediadas por las tecnologías informáticas. Tecnologíaseducativas. 2(2).

Silva, A. (2015). Softareas para contribuir al desarrollo de habilidades en la solución de ecuaciones lineales en los alumnos de 8vo grado de la ESBU "Lázaro Duarte Duarte" (doctoral dissertation, Universidad de Ciencias Pedagógicas “Félix Varela morales").

Soca Guevara, E. (2015). El trabajo independiente en el proceso de enseñanza-aprendizaje. Revista Cubana de Informática Médica, 15(2), 122-131.

Tesipov, V. (1981). El trabajo independiente de los alumnos en las clases. Moscú. Ed. Utshpedguis, 15-35. 